\title{
Etat Des Lieux Du Dispositif D'alimentation Dans Quelques Écoles Primaires De La Ville De Yaoundé (Cameroun)
}

\section{Annie-Claude Pial \\ Malika Mboupaing}

Laboratoire des Biotechnologies Végétales et Environnement, Département de Biologie et Physiologie Végétales, Faculté des Sciences, Université de

Yaoundé I, BP 812 Yaoundé, Cameroun.

\section{Majesté Mbiada Pahane}

Laboratoire de Microbiologie et Biotechnologie Alimentaires, Département des Sciences alimentaires et Nutrition, Ecole Nationale Supérieure des

Sciences Agro-Industrielles, Université de Ngaoundéré, BP 455 Ngaoundéré, Cameroun.

\section{Germain Ndengue}

Laboratoire des Biotechnologies Végétales et Environnement, Département de Biologie et Physiologie Végétales, Faculté des Sciences, Université de Yaoundé I, BP 812 Yaoundé, Cameroun.

\begin{abstract}
School feeding refers to all the conditions through which pupils have access, choose and consume food within the school. School feeding has gained increasing attention in recent years and it is an important route in the process for achieving quality education. The availability of healthy and balanced food in schools is a necessity highlighted in many studies. In developed countries, the institutional framework for school feeding is well developed, schools have canteens, where the meals are prepared and consumed by children according to health standards. The situation is different in developing countries, especially in Cameroon where we carried out a study with an aim to have an overview of the feeding organization in some primary schools in the Yaounde I subdivision. The methodology adopted consist of a survey carried out amongst school stakeholders (school principals, teachers, students and food handlers) and visits to targeted schools. According to the results obtained, $85 \%$ of the targeted schools don't have a building space, equipped with all the amenities (Clean water, tables, chairs, toilets) and ready to receive food vendors. Different types of foods
\end{abstract}


are served to children (meals, pastries, fruits, sweets, beverages and icecreams). Unfortunately, those foods are prepared and serve under low hygienic conditions according to standards required for this purpose. Many deficiencies have been highlighted in the framework of our study and in order to mitigate them, the national government should take some actions such as the development of a national policy, the establishment of an adequate institutional framework, the development of a sustainable financing strategy and a capacity building strategy food handlers.

Keywords: School feeding, organization, Yaounde, Cameroon

\section{Resume}

L'alimentation en milieu scolaire fait l'objet d'un intérêt accru ces dernières années et se positionne comme un levier à actionner pour atteindre les objectifs d'une éducation de qualité. La disponibilité d'aliments sains et équilibrés dans les écoles est une nécessité mise en exergue dans de nombreux travaux. L'étude que nous avons menée avait pour objectif de faire l'état des lieux du système d'alimentation dans quelques écoles primaires de l'arrondissement de Yaoundé $\mathrm{I}^{\mathrm{er}}$. La méthodologie adoptée s'est articulée autour des trames d'enquêtes soumises aux intervenants de la chaine de l'alimentation scolaire (Directeurs d'écoles, enseignants, élèves et vendeurs) et des visites dans les écoles. Il ressort des résultats obtenus que $85 \%$ des écoles n'ont pas de bâtiment formellement construit, muni de toutes les commodités et prêts à accueillir les vendeuses et vendeurs. Ces derniers proposent des aliments variés (plats cuisinés, pâtisseries, fruits, confiseries boissons et glaces) qu'ils apprêtent et servent dans des conditions hygiéniques nettement en dessous des normes exigibles en la matière. En conclusion, il apparaît clairement que le secteur de l'alimentation scolaire présente de nombreuses insuffisances qu'il est souhaitable d'adresser. L'élaboration d'une politique nationale, d'un cadre institutionnel adéquat, d'un financement durable et le renforcement des capacités des acteurs impliqués dans la production et la vente des aliments dans les écoles seraient des étapes essentielles à franchir pour améliorer cette situation.

Mots clés : Dispositif, Alimentation scolaire, Yaoundé, Cameroun

\section{Introduction}

L'alimentation scolaire désigne l'ensemble des conditions dans lesquelles les élèves ont accès aux aliments, les choisissent et les consomment à l'intérieur de l'école. Elle fait l'objet d'un intérêt accru ces dernières années et se positionne comme un levier à actionner pour atteindre les objectifs d'une éducation de qualité. La disponibilité d'une alimentation 
saine et équilibrée dans les écoles, préalable au maintien des enfants en bonne santé est une nécessité mise en exergue par de nombreuses études (Lawson, 2012 ; Jomaa et al., 2014 ; Oot et al., 2016). Ces dernières établissent le lien entre la nutrition et le développement cognitif et étayent l'assertion selon laquelle une mauvaise alimentation est associée à de moins bons résultats d'apprentissage. Dans les pays développés le cadre institutionnel organisant l'alimentation scolaire est bien élaboré (PAM, 2013), les écoles possèdent des cantines, espaces dans lesquelles les repas consommés par les élèves sont confectionnés dans des conditions conformes aux standards sanitaires. Ce n'est pas le cas au Cameroun et dans bien d'autres pays en développement où la majorité des écoles n'ont pas de cantines scolaires (Yunusa et al., 2012: Diarra, 2015). Le secteur de l'alimentation scolaire est par conséquent détenu par des micro-entreprises qui produisent des aliments et des boissons vendus et consommés dans et aux alentours des écoles. Malgré le fait que l'alimentation scolaire soit un maillon essentiel du dispositif éducatif (PAM, 2013), il n'a jusqu'à présent pas fait l'objet au Cameroun, d'études approfondies dans la perspective de mieux cerner sa structuration et de ressortir les problèmes spécifiques auxquels il est confronté. C'est la faiblesse que se propose d'adresser le présent travail de recherche dont l'objectif est de faire l'état des lieux du dispositif d'alimentation dans quelques écoles primaires de l'arrondissement de Yaoundé $\mathrm{I}^{\mathrm{er}}$. Les résultats de cette étude constitueront des données de référence à partir desquelles des stratégies seront formulées par les décideurs afin de mieux organiser ce secteur.

\section{Materiel et methodes Site d'étude}

L'étude avait pour cadre l'arrondissement de Yaoundé $\mathrm{I}^{\mathrm{er}}$, l'un des sept que compte la capitale politique du Cameroun. Chef-lieu de la région du centre et du département du Mfoundi, Yaoundé est située à environ $260 \mathrm{~km}$ de la côte Atlantique, à $3^{\circ} 52^{\prime}$ de latitude nord et $16^{\circ} 04^{\prime}$ 'de longitude Est et abrite les institutions les plus importantes du pays. Le relief accidenté, est dominé par des collines dont l'altitude moyenne est de $800 \mathrm{~m}$. Le climat est de type équatorial à quatre saisons, 2 saisons des pluies et 2 saisons sèches (petites et grandes), la pluviométrie oscille autour de $1650 \mathrm{~mm} / \mathrm{an}$ et la température moyenne est de $23,5^{\circ} \mathrm{C}$. La végétation est du type intertropical avec prédominance de la forêt humide méridionale. 


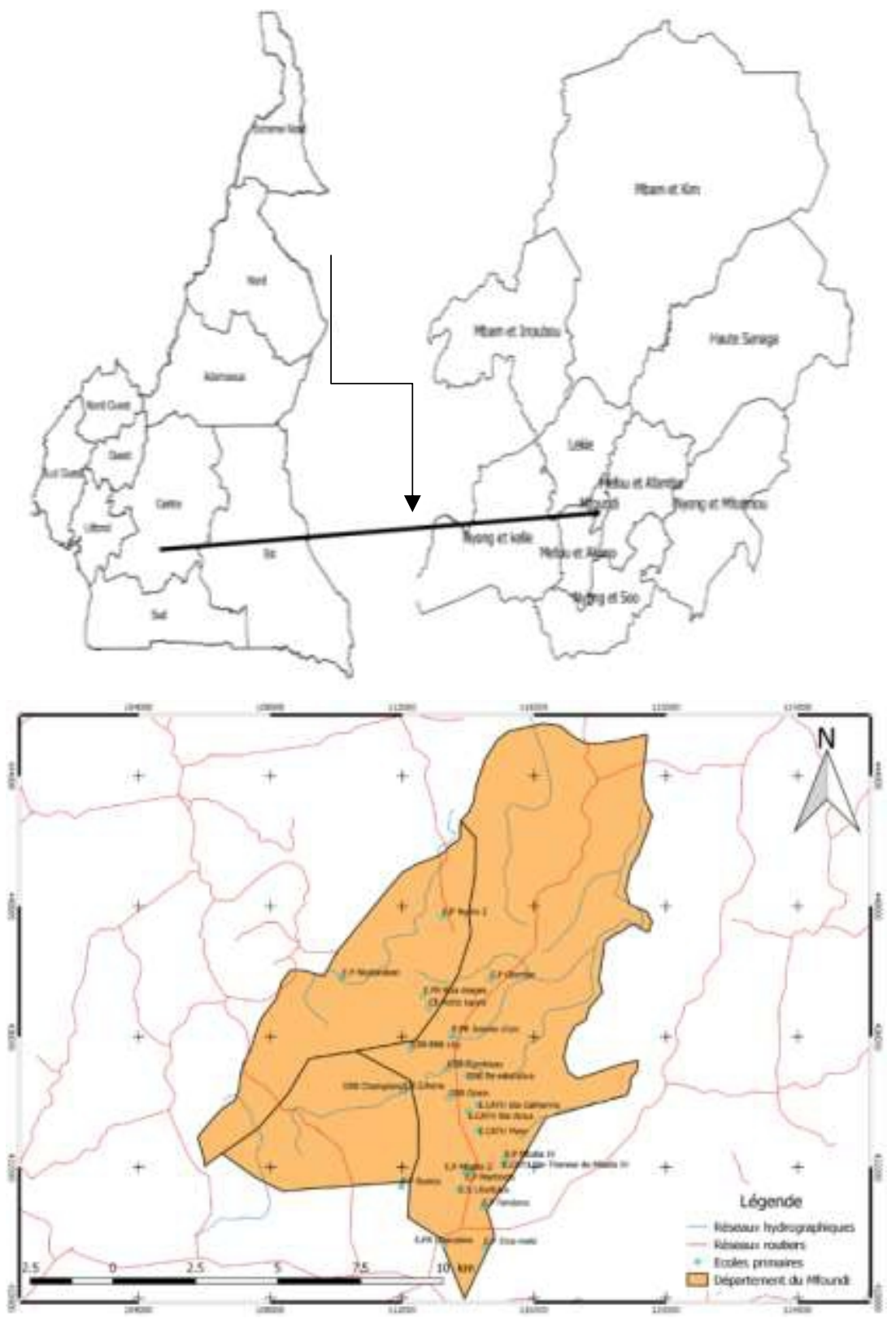

Fig.1. Localisation géographique du site d'étude

\section{Collecte des données}

Les écoles situées dans l'arrondissement de Yaoundé $\mathrm{I}^{\mathrm{er}}$ ont été sélectionnées selon la méthode aléatoire simple. Les critères qui ont prévalu sont : l'effectif, l'accessibilité, la présence d'un point de ravitaillement et la réceptivité du staff dirigeant. Sur la base de ces critères, 30 écoles primaires publiques ont été sélectionnées sur les 45 que compte cet arrondissement. 
Après obtention des autorisations administratives, des séances de prise de contact ont été organisées avec les staffs dirigeants dans l'optique de présenter les objectifs de l'étude, d'échanger sur la méthodologie et d'élaborer de manière consensuelle le programme des descentes dans les écoles.

Dans chaque école, deux types d'outils ont été utilisés :

les trames d'enquêtes réalisées à l'aide du logiciel Sphinx Plus2 Edition lexica-V5 ont été soumises aux Directeurs (25), enseignants (120), élèves (300) et vendeurs (200). Elles ont renseigné sur les modalités d'installation des vendeuses, les conditions d'hygiène qui leur sont imposées, les instructions données aux élèves et la perception que ces derniers ont des repas qui leur sont vendus.

les observations directes ont permis d'apprécier la conformité des informations reçues.

\section{Analyse des résultats}

Les données issues des enquêtes ont été dépouillées manuellement, codifiées, saisies, traitées et analysées au moyen du logiciel d'analyse statistique Sphinx.

\section{Resultats}

\section{Caractéristiques des zones de vente}

Abusivement appelées cantine dans la majorité des écoles, les zones de vente des aliments sont situées à certains coins des cours des écoles et servent de lieux de ravitaillement des élèves pendant les récréations. Dans $15 \%$ des écoles, ce sont des hangars formellement construits, couverts de tôles tandis que dans $85 \%$, les vendeuses et vendeurs sont regroupés à l'air libre. $75 \%$ des vendeurs et vendeuses présentent leurs produits sur des étales construites en bois alors que $25 \%$ étalent leurs produits sur des nattes de fortune, à même le sol. Dans aucune des écoles ciblées par notre étude, on a identifié à proximité de ces zones de vente un dispositif d'approvisionnement en eau pour faciliter l'entretien des ustensiles de service.
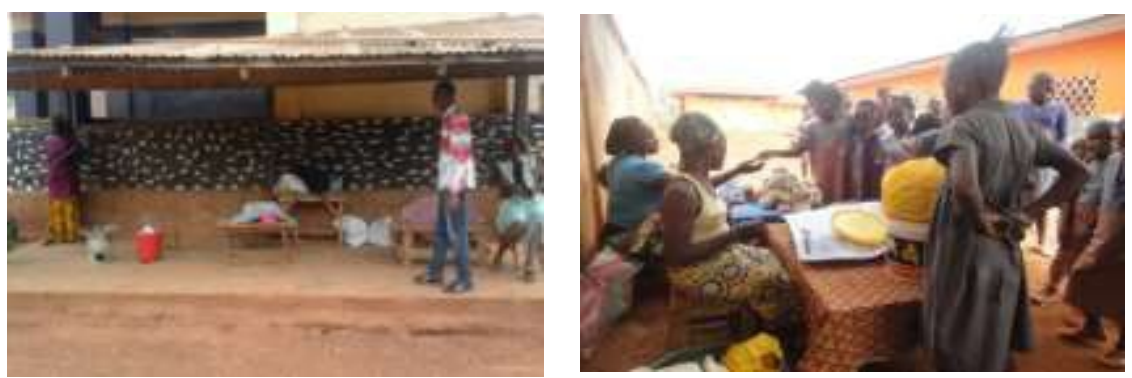

Photo 1. Zones d'approvisionnement des élèves 
Ces zones de vente sont à $96 \%$ occupées par des vendeuses, les vendeurs ne représentant que $4 \%$. Les vendeuses et vendeurs ont tous reçu une autorisation de la direction de l'école raison pour laquelle ils exercent leur activité dans les enceintes. Ces autorisations sont délivrées au terme de l'étude d'un dossier constitué d'une demande, d'un certificat médical et d'une photocopie de la carte nationale d'identité. Les vendeurs sélectionnés se doivent de respecter les prescriptions édictées par la direction. Il s'agit de la présentation d'un certificat médical tous les trois mois, le port obligatoire d'une blouse blanche et d'un bonnet blancs et l'entretien des espaces de vente. Ces règles ne sont malheureusement pas consignées dans un document signé par les vendeuses et vendeurs, ce qui rend aléatoire leur application.

Les vendeuses et vendeurs ont un niveau éducatif moyen comme le démontre la figure ci-dessous. 25 n'ont pas fini le cycle primaire; 99 ont le diplôme sanctionnant la fin du cycle primaire, le CEPE (Certificat d'Etudes Primaires Elémentaires) ; 63 ont le diplôme sanctionnant la fin du premier cycle du secondaire, le BEPC et seulement 13 ont plus que le BEPC. De tels niveaux d'étude les prédisposent à comprendre et intégrer la nécessité de prendre en compte les règles d'hygiène essentielles au bon déroulement de leur activité. Malheureusement, aucune initiative visant à renforcer leurs capacités n'est initiée dans les différentes écoles.

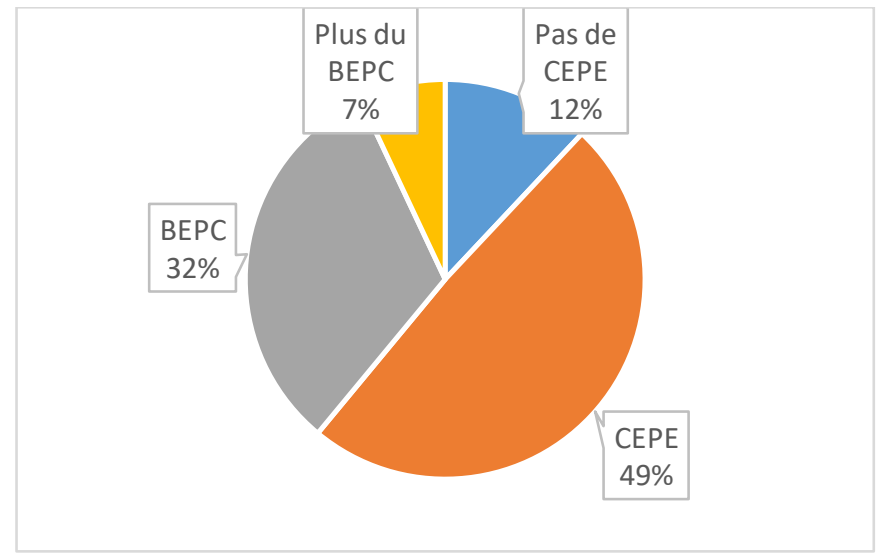

Fig. 2. Niveau éducatif des vendeuses et vendeurs

\section{Identification des aliments}

Les aliments vendus dans les différentes écoles sont de nature variée. Aucune instruction d'un diététicien n'est obtenue en rapport avec le choix des aliments à proposer aux élèves. Plusieurs catégories d'aliments ont été identifiés (les plats cuisinés, les pâtisseries, les légumes, les confiseries les boissons et glaces).

Tableau I. Classification des aliments vendus dans les écoles 


\begin{tabular}{|c|c|}
\hline Plats cuisinés & $\begin{array}{c}\text { Riz, éru, haricot, spaghetti, poisson haché (Sardine et thon), viande } \\
\text { hachée, purée, œufs, bouillie }\end{array}$ \\
\hline Pâtisseries & Pain, gâteau, beignet \\
\hline Fruits & En fonction des saisons (Papaye, orange, goyave, pastèque) \\
\hline Confiseries & Bonbons, biscuits, maïs grillé, caramel \\
\hline Boissons & Foléré, jus de fruits, sucette, eau en sachet \\
\hline Glaces & Vanille, chocolat et saveurs diverses \\
\hline
\end{tabular}

Sur les 200 vendeuses et vendeurs à qui le questionnaire a été soumis, 87 des vendeuses et vendeurs présentent des plats cuisinés sur leurs étalages, 48 de la pâtisserie, 37 des confiseries, 21 des boissons et 7 des fruits. On observe que contrairement aux informations données par le staff académique, selon lesquelles les vendeuses et vendeurs ont des autorisations pour un seul type d'aliments, cette consigne n'est pas respectée et ces derniers s'impliquent dans la vente de plusieurs autres produits dont en l'occurrence les boissons (eau en sachet et jus naturels).

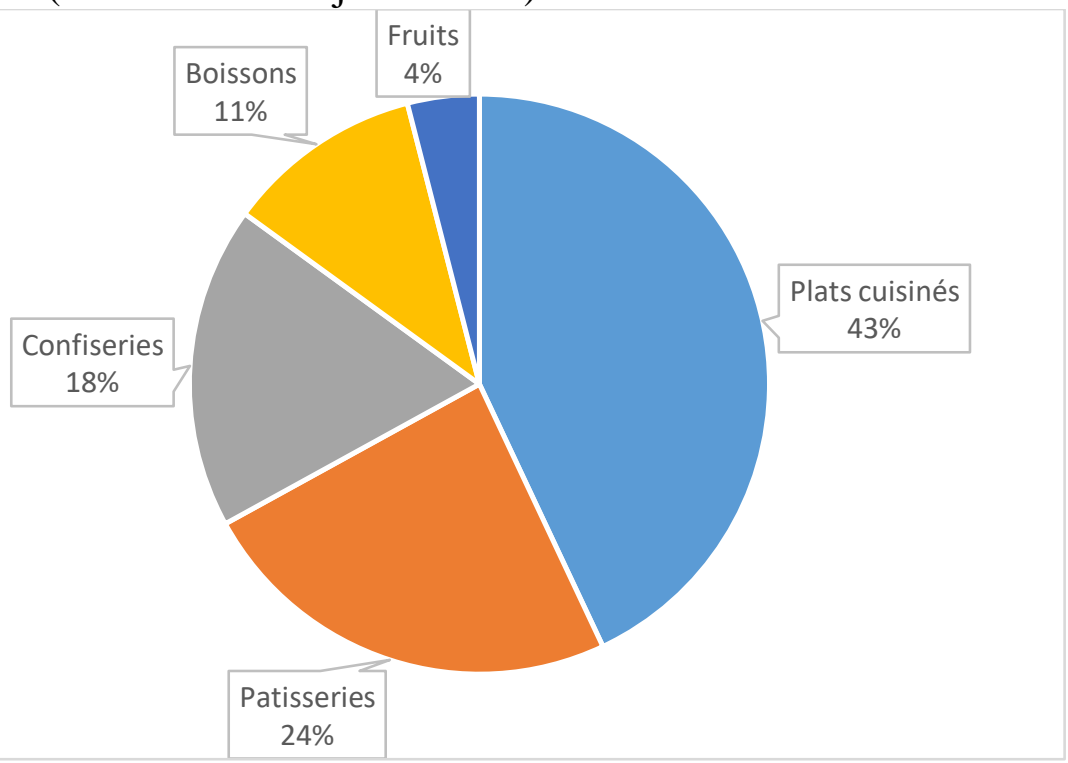

Fig. 3. Proportions des aliments proposés aux élèves

Les plats cuisinés sont majoritairement vendus aux élèves sous forme de sandwich qui se décline sous plusieurs types, à des prix qui varient de 100 frs CFA à 250 frs CFA en fonction de la longueur du pain, de la qualité (viande hachée, poisson haché, œufs bouillis ou omelette) et du volume de son contenu. Parce que c'est un aliment composé dont la préparation se fait 
en plusieurs étapes, le sandwich est sujet à la contamination bactérienne avec des conséquences plus ou moins graves. Les vendeuses et vendeurs ne tiennent pas compte dans leur choix des besoins journaliers moyens d'énergies et de protéines de la population scolaire tels que décrit dans le Manuel d'alimentation scolaire (PAM, UNESCO et OMS, 1999).

\section{Respect des règles d'hygiène}

Dans la majorité des écoles, les espaces de vente des aliments sont bien entretenus par les vendeuses et vendeurs qui se chargent de leur entretien au quotidien suivant un calendrier hebdomadaire, arrêté par eux même de manière consensuelle. C'est par ailleurs une exigence des directions des écoles qui désignent de façon hebdomadaire des maîtres et maîtresses de service pour assurer les inspections.

Certains aliments sont présentés sur des étalages à la hauteur exigée de $50 \mathrm{~cm} \mathrm{du} \mathrm{sol.} \mathrm{D'autres} \mathrm{par} \mathrm{contre} \mathrm{le} \mathrm{sont} \mathrm{à} \mathrm{même} \mathrm{le} \mathrm{sol,} \mathrm{sur} \mathrm{des} \mathrm{nattes} \mathrm{de}$ fortune. Les aliments sont pour certains, conservés dans des seaux en plastique transparents, des marmites avec couvercles et des thermos. D'autres par contre sont exposés sur des plateaux à l'air libre, sujets aux intempéries et aux contaminations.
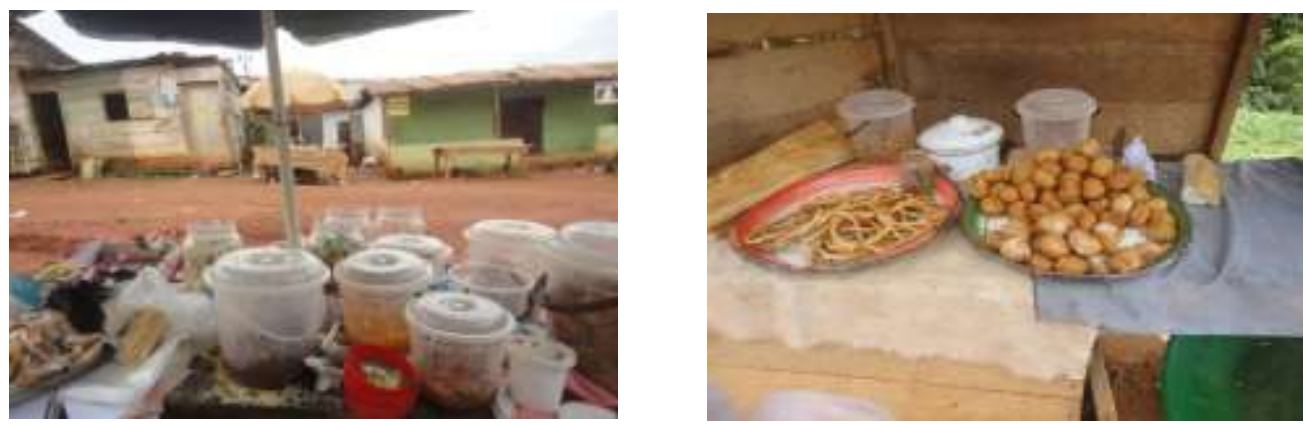

Photo 2. Exposition et de conservation des aliments

Plus de la moitié des élèves se ravitaillent auprès des vendeuses et vendeurs installés dans les cours de récréation. Plusieurs raisons sous-tendent ce choix : ils habitent loin de l'école, ne prennent pas de petit déjeuné, n'ont pas de gouter, préfèrent les aliments proposés à l'école etc.). Le reste ramène leurs repas de la maison ou ne mangent pas à l'école pour des raisons variées. 


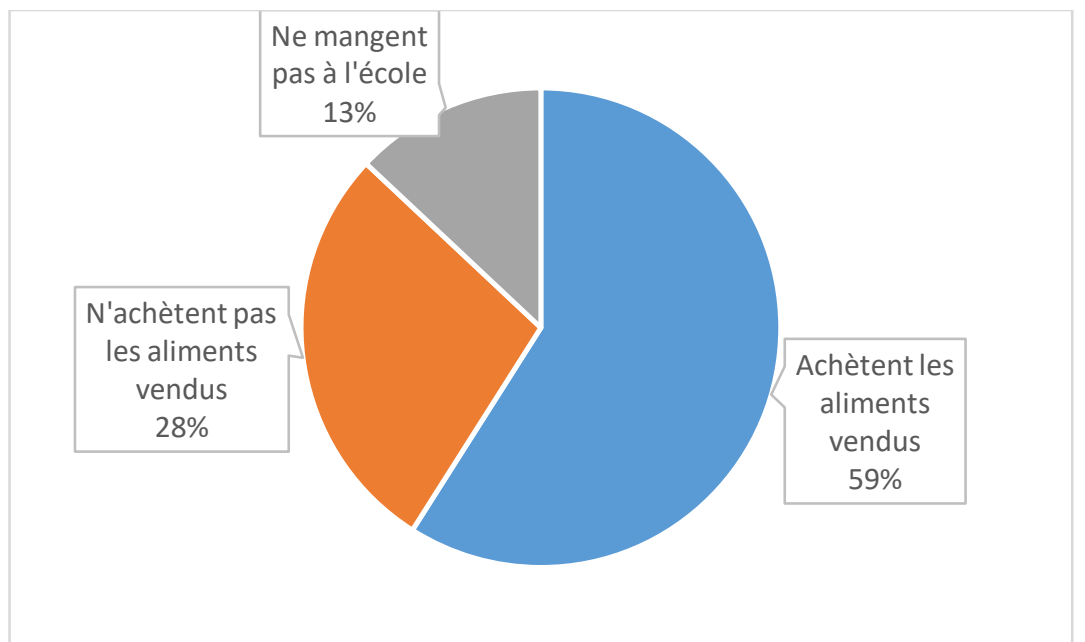

Fig. 4. Comportement des élèves par rapport aux aliments vendus

$100 \%$ des enseignants interrogés donnent des enseignements relatifs à la promotion des règles d'hygiène. Les élèves sont ainsi sensibilisés au lavage des mains avant les repas. Il se trouve cependant que $86 \%$ des élèves qui s'approvisionnent auprès des vendeuses et vendeurs n'appliquent pas cette règle c'est-à-dire ne prennent pas la peine de laver leurs mains avant les repas pris lors des recréations à cause de l'inexistante d'une source d'approvisionnement en eau à proximité et des pénuries d'eau fréquentes. $14 \%$ des élèves trouvent le moyen de se laver les mains avant de manger en utilisant l'eau stockée dans les salles de classe ou rapportée de leurs domiciles. La faible pratique de l'hygiène des mains par les élèves est une porte d'entrée aux contaminations de toutes sortes et pourrait avoir des répercussions sur l'absentéisme scolaire.

Malgré que les vendeuses et vendeurs aient dans leur grande majorité achevé le cycle primaire, ce qui laisse supposer qu'ils sont sensibilisés à l'importance de la pratique des règles d'hygiène, on observe par exemple que les conditions vestimentaires exigées pour exercer l'activité de vente ne sont pas respectées. 7\% seulement portent la blouse de couleur blanche exigée, $70 \%$ ne portent pas de bonnets ni foulards pour éviter la contamination des aliments. Les enquêtes menées indiquent par ailleurs qu'ils ne se soumettent pas fréquemment (tous les trois mois) aux contrôles médicaux qui permettent d'assurer qu'ils ne sont pas porteurs de germes transmissibles. Par ailleurs toutes les vendeuses et vendeurs ne protègent pas les aliments qu'ils vendent contre la poussière et les insectes vecteurs de maladies. Une fois vendus, les aliments sont emballés avec du papier usagé ou encore avec des sachets plastique réutilisés. Le service se fait à mains nues et aucune assurance relative au lavage des mains avant le début du service ne peut être garantie. 
Les aliments vendus dans les écoles sont achetés dans les marchés de la ville sans que les aspects fraicheurs ne soient particulièrement pris en compte. 92\% sont préparés dans les domiciles respectifs des vendeuses et vendeurs la veille, puis acheminés par un moyen de transport (taxi, poussepousse) au lieu de vente. Les directions des écoles n'ont par conséquent aucune possibilité de contrôle de la qualité des aliments proposés. 8\% des vendeuses préparent leurs aliments sur place, à l'air libre grâce à des réchauds à pétrole montés sur des pousse-pousses ou des brouettes. Même préparés sur place, des doutes subsistent sur la qualité microbiologique des aliments.

\section{Discussions}

Compte tenu de l'impact avéré de l'alimentation scolaire dans l'amélioration des résultats des apprenants, elle est devenue une question émergente majeure pour la promotion d'une éducation de qualité. C'est ce qui justifie l'institution en 2016, d'une journée de l'alimentation scolaire par la Commission de l'Union Africaine.

$\mathrm{Au}$ Cameroun, à l'exception de quelques localités situées dans les parties septentrionales, dans lesquelles des programmes d'alimentation scolaire sont pilotés par le Programme Alimentaire Mondial (PAM), soutenu par des Organisations Non Gouvernementales (ONG) locales et internationales, l'alimentation scolaire est majoritairement l'œuvre des micro-entreprises locales. Cette option a l'avantage de la rendre moins dépendante des ressources externes et plus centrée sur la consommation de denrées localement produites. L'étude menée a permis de faire un état des lieux du dispositif d'alimentation scolaire dans quelques écoles primaires de l'arrondissement de Yaoundé I ${ }^{\mathrm{er}}$. Elle s'est appuyée sur la consultation des intervenants directs dans la gestion quotidienne de l'alimentation scolaire (Directeurs, maîtresses et maîtres, élèves, vendeuses et vendeurs) et les observations directes effectuées dans les écoles.

Les résultats obtenus indiquent qu'aucune des écoles ciblées ne dispose d'une cantine scolaire, c'est-à-dire d'un espace où les aliments sont préparés et servis dans des conditions sanitaires optimales et au sein duquel les élèves bénéficient de toutes les commodités (Chaises, tables, couverts, facilités pour le lavage des mains ...) pour prendre leurs repas. Les espaces de vente situés dans les cours de récréation, où se concentrent les vendeuses et vendeurs proposant des aliments prêts à la consommation sont de façon abusive appelées cantines. Ces espaces sont utiles parce qu'ils permettent aux élèves de répondre à leurs besoins alimentaires sans sortir de l'enceinte de l'école pendant les récréations et contribuent à atténuer le risque de déperdition scolaire, suite à l'abandon des cours après les pauses. Parce que ces espaces ne comportent pas toutes les commodités essentielles, la 
construction des cantines dans toutes les écoles devient une nécessité. Il serait souhaitable qu'elles soient en matériaux définitifs, avec des espaces conviviaux réservés à la prise des repas et des sources d'approvisionnement en eau pour maximiser la pratique des règles d'hygiène. Un tel investissement ne serait cependant pas réalisable à court terme au regard du budget annuel (5\% du budget national), alloué au Ministère en charge de l'Education de base. Une meilleure planification des investissements, un plaidoyer continu au niveau des instances de décision budgétaires et la recherche de partenariat avec le secteur privé permettraient à long terme d'atteindre l'objectif d'une cantine par école.

Les aliments vendus dans les différentes écoles sont de nature variée. On distingue : les plats cuisinés, les pâtisseries, les légumes, les confiseries, les boissons et glaces diverses. Les élèves ont besoin d'une alimentation favorisant une croissance harmonieuse et un développement optimal. Les aliments proposés participent certainement à la couverture de ces besoins et sont en cohérence avec les habitudes alimentaires camerounaises comme l'exige le PAM, l'UNESCO et l'OMS dans le manuel de l'alimentation scolaire publié en 1999. Il faut cependant noter que ces aliments n'ont pas été proposés par un diététicien ou encore par le service de la santé scolaire. Leur choix n'est pas non plus basé sur les principes qui guident la composition et le volume de la ration alimentaire à savoir 2000 kilocalories et $40 \mathrm{~g}$ de protéines pour les enfants de 6 à 12 ans (PAM, 1999). Le choix des aliments incombe entièrement aux vendeuses et vendeurs qui cherchent à faire le minimum d'investissement pour un maximum de bénéfice et ceci au détriment des aspects qualité. La non prise en compte des critères qui guident le choix des aliments vendus dans les écoles ciblées n'est pas une situation singulière. Des études menées au Mali (Diarra et al., 2015), dans 12 pays (Aliyar et al., 2015) dont au le Ghana, le Kenya, le Mali, le Rwanda et l'Inde révèlent toutes que les aspects liés à la valeur nutritive des aliments ne sont pas pris en compte lors de l'élaboration des menus, par les intervenants dans la vente des aliments dans les écoles.

Les aliments vendus dans les écoles sont dans leur grande majorité préparés dans les domiciles des vendeuses et vendeurs. Les enquêtes menées auprès de ces derniers permettent de les rattacher aux couches socioéconomiques défavorisées. Par conséquent, leurs habitations n'offrent pas toutes les commodités essentielles à la conduite d'une activité de restauration. A cause du mauvais entreposage des denrées dans les domiciles, de la préparation des aliments plusieurs heures avant leur consommation, de leur conservation à des températures inadéquates, de leur réchauffage lorsqu'ils n'ont pas été consommés, de la contamination croisée et la mauvaise hygiène personnelle de ceux qui manipulent les aliments, une forte suspicion pèse sur la qualité de ces aliments réf. Il faut ajouter à cela, le 
laxisme observé en termes de fréquence et d'efficience des contrôles exercés par les autorités éducatives. La qualité hygiénique des aliments vendus dans les rues reste très douteuse en Afrique et les résultats de cette étude ne sont pas marginaux. Ils sont en effet similaires à ceux d'études réalisées en Afrique du Sud (Ntow et al., 2016), en Ethiopie (Mulugeta et al., 2012), au Ghana (Atta et al., 2015 ; Nartey et al., 2017), au Kenya (Nyamari, 2013), au Maroc (Al Gheza et al., 2017) et au Nigéria (Ifeadike et al., 2014 ; Tolulope et al., 2015), qui mettent toutes en évidence des faiblesses dans le respect des règles d'hygiène dans le secteur de l'alimentation scolaire.

\section{Conclusion}

Le secteur de l'alimentation scolaire est considéré comme un puissant levier à actionner pour atteindre les Objectifs de Développement Durable (ODD), notamment ceux relatifs à la lutte contre la pauvreté et la faim (ODD 1 et 2), à la santé (ODD 3), à la promotion de l'éducation (ODD 4). L'étude menée dans 30 écoles primaires permet montre au goût du jour que ce secteur est encore caractérisé par l'amateurisme de ses acteurs et par l'inexistence d'une véritable structuration infrastructurelle et organisationnelle. Pour agir de façon intégrée sur ce déterminant d'un enseignement de qualité, le gouvernement doit prendre et mettre en œuvre un certain nombre de dispositions en faveur de l'alimentation scolaire dont en particulier : l'élaboration d'une politique nationale, d'un cadre institutionnel adéquat, prenant en compte les différents intervenants à tous les niveaux et favorisant une coordination cohérente, la mise à disposition d'un financement durable et stable pour améliorer le cadre infrastructurel et l'organisation régulière de sessions de renforcement des capacités destinées aux personnes impliquées dans la production et la vente des aliments vendus dans les écoles.

\section{References:}

1. Aliyar R., Gelli A., Hamdani S. H. (2015). A review of nutritional guidelines and menu compositions for school feeding programs in 12 countries. Frontiers in Public Health, 3(148): 13p. https://doi.org/10.3389/fpubh.2015.00148.

2. Atta G. P., Manu J. (2015). Ghana School Feeding Program: A Retrospective Review. International Journal of Innovative Research and Development, 4 (8): 402-410

3. Agyei-Baffour P., Boateng Sekyere K., Akosua Addy E. (2013). Policy on Hazard Analysis and Critical Control Point (HACCP) and adherence to food preparation guidelines: a cross sectional survey of stakeholders in food service in Kumasi, Ghana. BMC Research Notes $6: 442$. 
4. Cisse D., Traore M S., Coulibaly M. (2015). L'offre alimentaire dans les établissements scolaires du quartier de Sabalibougou en commune $V d u$ district de Bamako. Mali medical, tome $\operatorname{xxx} \mathrm{n}^{\circ} 2,7 \mathrm{p}$.

5. El Ghaza S., Chbab Y., Bakrim E., Zaadoud B., Chaouch A. (2017). Evaluation du système d'hygiène dans les locaux des restaurations scolaires, a la région d'El Gharb Cherardabnihssen, par rapport aux exigences normatives internationales. European Scientific Journal, 13 (2) : 243-255. Doi : 10.19044/esj.2016.v13n2p243

6. Ifeadike CO, Ironkwe OC, Adogu PO, Nnebue CC. (2014). Assessment of the food hygiene practices of food handlers in the Federal Capital Territory of Nigeria. Tropical Journal of Medical Reseacrh, 17:10-5.

7. Jomaa LH, McDonnell E., Probart C. (2014). School feeding programs in developing countries: impacts on children's health and educational outcomes. Nutrition Review, 69 (2): 83-98. DOI: https://doi.org/10.1111/j.1753-4887.2010.00369.x

8. Lawson T. M. (2012). Impact of school feeding programs on educational, nutritional, and agricultural development goals: a systematic review of literature. Master of science, Michigan State University, USA.

9. Mulugeta K., Bayeh A. (2012). The sanitary conditions of food service establishments and food safety knowledge and practices of food handlers in Bahir Dar town. Ethiopian Journal of Health Sciences, 22 (1): 27-35

10. Nartey, E., Owusu, J., Gamor, E., and Mensah, E. E. (2014). Assessment of Knowledge and Practices of Food Hygiene and Safety of Caterers inj Senior High Schools in Ghana: A Case Study of Two Senior High Schools in Koforidua. ADRRI Journal of Agriculture and Food Sciences, 3(2): 1-15.

11. Ntow G. E., Kwabla M. P., Der J. (2016). Hygiene Practices Among Food Vendors in Hohoe Township. Central African Journal of Public Health. 2 (2):43-50. doi: 10.11648/j.cajph.20160202.11

12. Tolulope O. A., Zuwaira I. H., Danjuma A. B., 1, Zaman M. (2015). Knowledge and practice of food safety and hygiene among food vendors in primary schools in Jos, Plateau State, North Central Nigeria. E3 Journal of Medical Research, 4(2): 016-022

13. Yunusa I., Gumel A. M., Adegbusi K., Adegbusi S. (2012). School feeding program in Nigeria: a vehicle for nourishment of pupils. The African Symposium. An online journal of the African Educational Research Network. 12 (2): 105-110. 
14. Nyamari J. (2013). Evaluation of compliance to food safety standards amongst food handlers in selected hospitals in kenya. Doctoral of philosophy, School of public health, Kenyatta University, 119p.

15. Oot L., Sethuraman K., Ross J., Sommerfelt A.E. (2016). The Effect of Chronic Malnutrition (Stunting) on Learning Ability, a Measure of Human Capital: A Model in Profiles for Country-Level Advocacy. Washington, DC: FHI 360/FANTA. 8p.

16. PAM, UNESCO, OMS (Programme alimentaire mondial, Organisation des Nations Unies pour l'Éducation, la Science et la Culture, Organisation mondiale de la Santé). (1999). Manuel d'alimentation scolaire. PAM, UNESCO, OMS.

17. PAM. (2013). La situation de l'alimentation scolaire dans le monde. Programme alimentaire mondial, Rome 00148, Italie. 125p. 